\title{
Anti-Glypican 3/CD3 Bispecific Antibody ERY974
}

National Cancer Institute

\section{Source}

National Cancer Institute. Anti-Glypican 3/CD3 Bispecific Antibody ERY974. NCI

Thesaurus. Code C129936.

An anti-glypican 3 (GPC3; GPC-3)/anti-CD3 bispecific monoclonal antibody, with potential immunostimulating and antineoplastic activities. Anti-GPC3/CD3 bispecific antibody ERY974 possesses two antigen-recognition and binding sites, one for the CD3 complex, a group of T-cell surface glycoproteins that complex with the T-cell receptor (TCR), and one for GPC3, a tumor-associated antigen (TAA) overexpressed on the surface of certain tumor cells. Upon administration of ERY974, this bispecific antibody simultaneously binds to both CD3-expressing and GPC3-expressing cells, thereby crosslinking GPC3expressing tumor cells and cytotoxic T-lymphocytes (CT Ls). This may result in potent CT L-mediated lysis of GPC3-expressing tumor cells. GPC3, a heparan sulfate proteoglycan and an oncofetal antigen protein, is overexpressed in a variety of cancers; it plays a role in cell division and growth regulation. 